\title{
On the Private Provision of Two or More Public Goods
}

\author{
RICHARD CORNES \\ Australian National University \\ JUN-ICHI ITAYA \\ Hokkaido University
}

\begin{abstract}
We extend the simple model of voluntary public good provision to allow for two or more public goods, and explore the new possibilities that arise in this setting. We show that, when there are many public goods, voluntary contribution equilibrium typically generates, not only too low a level of public good provision, but also the wrong mix of public goods. We also analyze the neutrality property in the more general setting, and extend a neutrality proposition of Bergstrom, Blume, and Varian (1986).
\end{abstract}

\section{Introduction}

Economists' intuitions concerning voluntary public good provision come mainly from the single public good model as set out, for example, by Cornes and Sandler (1985) and Bergstrom, Blume, and Varian (1986). The neutrality property and the conditions required for its validity are now well understood, as is the presumption that the voluntary contribution mechanism produces an inefficiently low equilibrium quantity of public good.

By contrast, there are few explicit analyses of voluntary contributions models with many public goods. Notable exceptions are Kemp (1984),

Richard Cornes, Research School of Economics, Australian National University, Canberra, ACT 0200, Australia (rccornes@aol.com). Jun-ichi Itaya, Graduate School of Economics and Business Administration, Hokkaido University, Sapporo, 060-0809, Japan (itaya@econ.hokudai.ac.jp).

Richard Cornes holds the F. H. Gruen Chair in Economics at the Australian National University. We are grateful to Indraneel Dasgupta, Albert Schweinberger, Jun Iritani, and Toshihiro Ihori for helpful comments on an earlier draft. Dasgupta and Kanbur (2005) have independently explored properties of models with many public goods. The usual disclaimer applies.

Received September 6, 2006; Accepted August 19, 2009.

(c) 2010 Wiley Periodicals, Inc.

Journal of Public Economic Theory, 12 (2), 2010, pp. 363-385. 
Bergstrom, Blume, and Varian (1986) and Cornes and Schweinberger (1996). Kemp establishes a neutrality proposition on the assumption that all players are positive contributors to every public good. Bergstrom, Blume, and Varian note that this assumption is problematic. They establish equilibrium existence in the presence of many public goods, and present a neutrality proposition. However, although they allow for the presence of noncontributors, their neutrality proposition invokes a rather restrictive assumption-one that can be relaxed, as we show below. Neither Kemp nor Bergstrom, Blume, and Varian address efficiency in the presence of many public goods. Cornes and Schweinberger simultaneously develop several extensions of the basic model, making it difficult to pinpoint precisely the implications of assuming many public goods.

There are good reasons for exploring models with two or more public goods. There are many examples of several public goods being voluntarily and simultaneously supplied in the real world-for example, many individuals contribute voluntarily to several charitable causes. If we take public goods seriously, our models should be able to accommodate the possibility of more than one. Not only may answers to existing questions change, but new questions arise as soon as a second public good is introduced.

In the standard model with a single public good, the possibility of corner solutions at which some individuals choose not to contribute must be taken seriously (see, e.g., Cornes and Sandler 1985, Bergstrom, Blume, and Varian 1986). However, such a situation, though likely, is by no means certain. A model with sufficiently few players may well have an equilibrium in which all choose to contribute. By contrast, if there is more than one public good, individuals will be at a corner solution with respect to at least one public good unless preferences are very special, even if the potential contributors are few. We show in Sections 4, 5.1, and 6 of this paper that Warr's neutrality property - the total provision of a public good remains unchanged with respect to a redistribution of income-may hold even if a redistribution of income is implemented between individuals who do not contribute to some public good.

Furthermore, such corner solutions in the presence of two or more public goods generate a second potential source of inefficiency associated with the voluntary contribution model that is conceptually distinct from the widely accepted tendency toward underprovision. Not only will aggregate public good provision tend to be "too low," but also the mix of public goods may well be "wrong." By this we simply mean that, starting from a Nash equilibrium, it is possible to achieve a Pareto superior allocation simply by reallocating resources among various public goods without increasing the aggregate level of resources devoted to the provision of public goods above its equilibrium level. This aspect is addressed in Subsections 3.2 and 5.2 below. 


\section{Constrained Pareto Efficiency with Many Public Goods and Individuals}

There are $n$ players, a single private good, and $m$ public goods. Individual $i$ 's preferences are represented by the utility function

$$
u_{i}=u_{i}\left(c_{i}, \mathbf{G}\right), i=1,2, \ldots, n,
$$

where $c_{i} \geqq 0$ is individual $i$ 's consumption of the private good, and $\mathbf{G}$ is the vector of total quantities of $m$ public goods: $\mathbf{G} \equiv\left(G_{1}, G_{2}, \ldots, G_{m}\right) \in$ $R_{+}^{m}$. We denote the vector $\left(c_{1}, c_{2}, \ldots, c_{n}\right) \in R_{+}^{n}$ by c. $u_{i}($.$) is strictly in-$ creasing in all arguments, strictly quasi-concave, and everywhere differentiable. It is also assumed that both goods are essential; i.e., $\lim _{c_{i} \rightarrow 0} \partial u_{i} / \partial c_{i}=\lim _{G_{j} \rightarrow 0} \partial u_{i} / \partial G_{j}=\infty$. Assuming a linear production frontier as in Bergstrom, Blume, and Varian (1986), the production of one unit of public good $j, j=1,2, \ldots, n$, requires one unit of the numeraire private good. The total amount of the private good initially available is $W \in R_{++}$:

$$
\sum_{i=1}^{n} c_{i}+\sum_{j=1}^{m} G_{j} \leqq W
$$

\subsection{Pareto Efficiency}

Consider the problem

$$
\operatorname{Max}_{\mathbf{c} \in \mathbf{R}_{+}^{n}, \mathbf{G} \in \mathbf{R}_{+}^{m}}\left\{u_{1}\left(c_{1}, \mathbf{G}\right) \mid \sum_{i=1}^{n} c_{i}+\sum_{j=1}^{m} G_{j} \leqq W, u_{i}\left(c_{i}, \mathbf{G}\right) \geq \bar{u}_{i}, i=2, \ldots, n\right\},
$$

where $\bar{u}_{i}$ represents the arbitrarily assigned target utility level of individual $i$. The Lagrangean for this problem is

$$
L=u_{1}\left(c_{1}, \mathbf{G}\right)-\lambda_{1}\left[\sum_{i=1}^{n} c_{i}+\sum_{j=1}^{m} G_{j}-W\right]-\sum_{i=2}^{n} \lambda_{i}\left[\bar{u}_{i}-u_{i}\left(c_{i}, \mathbf{G}\right)\right] .
$$

The first-order conditions include

$$
\begin{aligned}
\frac{\partial L}{\partial c_{1}} & =u_{1 c}-\lambda_{1}=0, \\
\frac{\partial L}{\partial c_{i}} & =-\lambda_{1}+\lambda_{i} u_{i c}=0, i=2, \ldots, n, \\
\frac{\partial L}{\partial G_{j}} & =u_{1 j}-\lambda_{1}+\sum_{i=2}^{n} \lambda_{i} u_{i j}=0, \quad j=1, \ldots, m,
\end{aligned}
$$

where $u_{i c} \equiv \partial u_{i} / \partial c_{i}$ and $u_{i j} \equiv \partial u_{i} / \partial G_{j}$. Elimination of the Lagrangean multipliers and rearrangement yields the Samuelson conditions, one for each 
public good:

$$
\sum_{i=1}^{n} \frac{u_{i j}}{u_{i c}}=1, \quad j=1, \ldots, m .
$$

Pareto efficiency requires the provision of each public good to be taken to the point where the marginal social benefit equals the marginal social cost.

\subsection{Constrained Pareto Efficiency}

In the single public good economy, comparison of Samuelson's condition with the Nash equilibrium conditions shows that, in general, too few resources are devoted to public good provision at a voluntary contribution equilibrium. This remains true in the presence of two or more public goods. However, we are interested in a further question: when there is more than one public good, what can we say about the mix of public goods? We will argue that Nash equilibrium may well also imply an inefficient mix of public goods in the following sense. Starting from an equilibrium, it is generally possible to reduce output of one public good and increase that of another so as to generate a Pareto improvement, even without increasing the aggregate level of resources devoted to public good production. To address this issue, we introduce the notion of constrained Pareto efficiency.

Definition 1: The allocation $\left(\mathbf{c}^{\prime}, \mathbf{G}^{\prime}\right) \in \mathbf{R}_{+}^{n} \times \mathbf{R}_{+}^{m}$ is "constrained Pareto efficient" if there exists no other feasible allocation $(\mathbf{c}, \mathbf{G}) \in \mathbf{R}_{+}^{n} \times \mathbf{R}_{+}^{m}$ such that

$$
\sum_{j=1}^{m} G_{j}=\sum_{j=1}^{m} G_{j}^{\prime}
$$

and

$$
u_{i}\left(c_{i}, \mathbf{G}\right) \geqq u_{i}\left(c_{i}^{\prime}, \mathbf{G}^{\prime}\right), \quad i=1,2, \ldots, n
$$

with at least one strict inequality. ${ }^{1}$

To characterize constrained Pareto-efficient allocations, we consider allocations consistent with the requirement that

$$
\sum_{j=1}^{m} G_{j}=K,
$$

\footnotetext{
${ }^{1}$ The constraint on public goods implies that an equal amount of resources is expended on producing the two profiles of public goods such as $\mathbf{G}, \mathbf{G}^{\prime} \in \mathbf{R}_{+}^{m}$. If the cost of producing one unit of public good $j$ in terms of the numeraire were constant at $p_{j}$, the constraint would read $\sum_{j=1}^{m} p_{j} G_{j}=\sum_{j=1}^{m} p_{j} G_{j}^{\prime}$.
} 
where $K \in R_{++}$is exogenously fixed. Within the set of feasible allocations in which the total cost of public good provision is constrained to equal $K$, a constrained Pareto-efficient allocation solves the problem:

$\operatorname{Max}_{\mathbf{c} \in \mathbf{R}_{+}^{n}, \mathbf{G} \in \mathbf{R}_{+}^{m}}\left\{u_{1}\left(c_{1}, \mathbf{G}\right) \mid \sum_{j=1}^{m} G_{j}=K, \sum_{i=1}^{n} c_{i}=W-K, u_{i}\left(c_{i}, \mathbf{G}\right) \geq \bar{u}_{i}, i=2, \ldots, n\right\}$.

The Lagrangean expression is

$$
\begin{aligned}
L= & u_{1}\left(c_{1}, \mathbf{G}\right)-\mu\left[\sum_{j=1}^{m} G_{j}-K\right]-\lambda_{1}\left[\sum_{i=1}^{n} c_{i}-W+K\right] \\
& -\sum_{i=2}^{n} \lambda_{i}\left[\bar{u}_{i}-u_{i}\left(c_{i}, \mathbf{G}\right)\right] .
\end{aligned}
$$

Standard manipulations yield

$$
\sum_{i=1}^{n} \frac{u_{i j}}{u_{i c}}=\sum_{i=1}^{n} \frac{u_{i k}}{u_{i c}}=\frac{\mu}{\lambda_{1}}, \quad j, k=1, \ldots, m ; j \neq k .
$$

PROPOSITION 1: Consider an economy with $n$ individuals, $m$ public goods, and a single private good. Assume that all utility functions are everywhere strictly quasiconcave, strictly increasing, and differentiable. Then, among allocations in which every individual consumes a strictly positive quantity of the private good, a necessary condition for constrained Pareto efficiency is that

$$
\sum_{i=1}^{n} \frac{u_{i j}}{u_{i c}}=\sum_{i=1}^{n} \frac{u_{i k}}{u_{i c}}, \quad j, k=1, \ldots, m ; j \neq k .
$$

Constrained Pareto efficiency is consistent with the aggregate level of resources to public good provision being "too high" (i.e., $\mu / \lambda_{1}<1$ ) or "too low" (i.e., $\mu / \lambda_{1}>1$ ), but insists that, whatever that level is, the sum of marginal benefits must be equal across all public goods. If this is not the case, it is possible to obtain a Pareto improvement while leaving the amount of resources available to public good production, $\sum_{j=1}^{m} G_{j}$, unchanged.

To see this, consider small perturbations from an arbitrary initial allocation that is not constrained Pareto efficient in an example with two individuals and two public goods. We have

$$
\begin{aligned}
d u_{i} & =u_{i c} d c_{i}+u_{i 1} d G_{1}+u_{i 2} d G_{2}, \quad i=1,2, \\
d c_{1}+d c_{2} & =0 \\
d G_{1}+d G_{2} & =0 .
\end{aligned}
$$


Consider perturbations consistent with $d u_{1}=0$. Substituting and rearranging,

$$
\left[\frac{d c_{1}}{d G_{1}}\right]_{d u_{1}=0}=\frac{u_{12}}{u_{1 c}}-\frac{u_{11}}{u_{1 c}}
$$

Similarly,

$$
\left[\frac{d c_{1}}{d G_{1}}\right]_{d u_{2}=0}=\frac{u_{21}}{u_{2 c}}-\frac{u_{22}}{u_{2 c}} .
$$

Pareto improvement is possible if and only if, in the neighborhood of the initial allocation,

$$
\left[\frac{d c_{1}}{d G_{1}}\right]_{d u_{1}=0} \neq\left[\frac{d c_{1}}{d G_{1}}\right]_{d u_{2}=0} .
$$

Using (6) and rearranging, Pareto improvement is possible if and only if

$$
\frac{u_{11}}{u_{1 c}}+\frac{u_{21}}{u_{2 c}} \neq \frac{u_{12}}{u_{1 c}}+\frac{u_{22}}{u_{2 c}},
$$

that is, if and only if (3) is violated.

We argue below that the inequality in (7) will generally hold at a Nash equilibrium. Equality is not implied by Nash equilibrium, and has zero probability. Hence, it is "almost always" possible to find a Pareto improving perturbation from an initial equilibrium allocation through a reallocation of expenditure between the public goods, accompanied by an appropriate reallocation of the resources available for private consumption. The nature of the reallocations required to generate Pareto improvement depends on the precise magnitudes of the responses (4) and (5).

\section{Equilibrium and Efficiency in a $2 \times 2$ Model}

\subsection{The Model}

This section works through a specific example that confirms that a Nash equilibrium is not typically constrained Pareto efficient. It also shows how different distributions of income across individuals affect the configuration of individual contributions to multiple public goods.

Although we first consider a model with two individuals and two public goods for expositional convenience (for which reason we will call this the $2 \times 2$ model), we will analyze the so-called $n \times m$ model involving $n(\geqq 2)$ individuals and $m(\geqq 2)$ goods later. Without loss of generality, we assume throughout the paper that the production costs of all goods per quantity are unity relative to the numeraire. Individual $i$ 's budget constraint is

$$
c_{i}+x_{i}+y_{i}=w_{i},
$$


where $x_{i}$ and $y_{i}$ represent individual $i$ 's contributions to the public goods $X$ and $Y$, respectively, and $w_{i}$ is her exogenous income. We use the notations $X$ and $Y$ to stand for the total quantities of these two types of public goods, respectively, in order to avoid double subscripts in the following sections (except for Section 6). Individual $i$ maximizes (1) by its choice of $c_{i}, x_{i}$, and $y_{i}$ subject to its budget constraint (8) and the non-negativity constraints $c_{i} \geqq 0, x_{i} \geq 0$, and $y_{i} \geq 0 .{ }^{2}$ As is standard in the literature on the private provision of public goods, this problem can be recast as a problem of choosing $c_{i}, X$, and $Y$, that is,

$$
\begin{aligned}
& \max _{c_{i}, X, Y} u_{i}\left(c_{i}, X, Y\right), \\
& \text { s.t. } c_{i}+X+Y=w_{i}+X_{-i}+Y_{-i}, \\
& X-X_{-i} \geqq 0 \quad \text { and } \quad Y-Y_{-i} \geq 0,
\end{aligned}
$$

where $X_{-i}$ and $Y_{-i}$, respectively, represent the contributions of everyone except $i$ to the public goods $X$ and $Y$ : thus $X=x_{i}+X_{-i}$ and $Y=y_{i}+Y_{-i}$.

Ignoring the non-negativity constraints and using the implicit function theorem, one can easily see that each individual has a solution to this problem of the functions $X_{i}^{*}\left(w_{i}+X_{-i}+Y_{-i}\right)$ and $Y_{i}^{*}\left(w_{i}+X_{-i}+Y_{-i}\right)$, or equivalently, $x_{i}=X_{i}^{*}\left(w_{i}+X_{-i}+Y_{-i}\right)-X_{-i}$ and $y_{i}=Y_{i}^{*}\left(w_{i}+X_{-i}+Y_{-i}\right)-Y_{-i}$. Taking into account the non-negativity constraints, we can, respectively, write the individual supply functions as

$$
\begin{aligned}
& x_{i}=\max \left\{X_{i}^{*}\left(w_{i}+X_{-i}+Y_{-i}\right)-X_{-i}, 0\right\} \text { and } \\
& y_{i}=\max \left\{Y_{i}^{*}\left(w_{i}+X_{-i}+Y_{-i}\right)-Y_{-i}, 0\right\} .
\end{aligned}
$$

Given these supply functions, we can define a Nash equilibrium as a vector of contributions $\left(x_{1}, y_{1}, x_{2}, y_{2}\right) \in R_{+}^{4}$ such that the supply functions in (9) can map this vector into itself. Under the assumptions made, it can easily be shown by adapting the standard existence proof used in the model of a single public good that a Nash equilibrium will exist.

It is easy to prove the following proposition:

PROPOSITION 2: In the $2 \times 2$ model with preferences described by (1),

(i) there "almost surely" does not exist a Nash equilibrium in which both players simultaneously make positive contributions to both public goods. However, if the preferences of both players are identical, then

(ii) there exists a Nash equilibrium in which both simultaneously make positive contributions to both public goods which is constrained Pareto efficient, and

\footnotetext{
${ }^{2}$ Since consumption of the private good always takes a positive value because of the essential good condition, $\lim _{c_{i} \rightarrow 0} \partial u_{i} / \partial c_{i}=\infty$, the non-negative constraint on $c_{i}$ is never binding.
} 
(iii) the profile of individual contributions to each public good is indeterminate at a Nash equilibrium.

Proof: Suppose that both simultaneously contribute to both public goods. The associated first-order conditions are given by

$$
\begin{gathered}
\operatorname{MRS}_{i}^{c X}\left(c_{i}, X, Y\right) \equiv \frac{\partial u_{i} / \partial X}{\partial u_{i} / \partial c_{i}}=1 \text { and } \operatorname{MRS}_{i}^{c Y}\left(c_{i}, X, Y\right) \equiv \frac{\partial u_{i} / \partial Y}{\partial u_{i} / \partial c_{i}}=1, \\
i=1,2 .
\end{gathered}
$$

Summing the budget constraint (8) over $i$ yields

$$
c_{1}+c_{2}+X+Y=w_{1}+w_{2} .
$$

Taken together, there are five equations that determine four unknown variables $\left(c_{1}, c_{2}, X, Y\right)$. There are no values of $\left(c_{1}, c_{2}, X, Y\right)$ that simultaneously satisfy the five equations (10) and (11) except for coincidence (such as identical preferences). This completes the proof of (i).

Under identical preferences, however, all of the first-order conditions in (10) can be consistent with each other. This is simply because the first-order conditions of both individuals in (10) are the same, and thus there are the only three equations involving the three variables $(c, X, Y)$ with $c \equiv c_{1}=c_{2}$. Hence, it is possible to determine the unique value of $(c, X, Y)$ satisfying these three equations. On the other hand, if $\left(x_{1}^{*}, x_{2}^{*}, y_{1}^{*}, y_{2}^{*}\right)$ is a Nash equilibrium vector of contributions, so too is any vector $\left(x_{1}^{*}+\Delta, x_{2}^{*}-\Delta, y_{1}^{*}-\Delta^{\prime}, y_{2}^{*}+\Delta^{\prime}\right)$ leaving the variables $(c, X, Y)$ unchanged, where $\Delta, \Delta^{\prime}$ may take any positive or negative value consistent with both individuals' budget constraints (8) being satisfied and contribution levels being non-negative, which completes the proof of (iii).

Finally, when the first-order conditions of both individuals are the same, (3) is automatically satisfied, and thus the Nash equilibrium is constrained Pareto efficient.

Proposition 1 implies that one or more of the first-order conditions for constrained Pareto efficiency does not hold with equality at a Nash equilibrium. If the inequality holds for some public good contributed by a particular individual, he or she stops contributing to that public good. Hence, at the Nash equilibrium of the model of multiple public goods there is always at least one individual who is a noncontributor to one public good. The absence of an interior Nash equilibrium in contribution space is mainly caused by a linear production technology (see Cornes and Schweinberger 1996 for a case of a nonlinear production technology). An exceptional case is that of identical preferences in which every individual can contribute to all public goods at a Nash equilibrium. In this case, even though the equilibrium levels of $X$ and $Y$ may be uniquely determined, individual contributions 


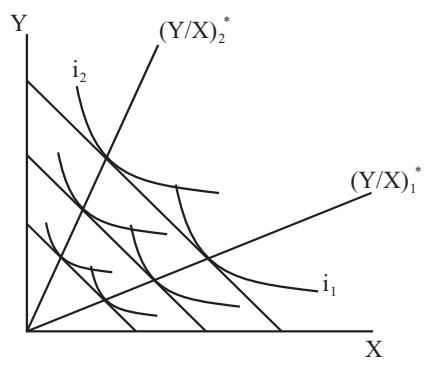

(a)

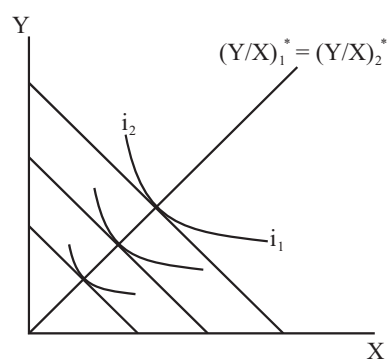

(b)

Figure 1: Individual i's expansion path.

are indeterminate, so that the individuals face a coordination problem with respect to their individual contribution levels. It should be borne in mind that the exact profile of individual contributions to multiple public goods is determined according to the structure of preferences as well as the profile of income distribution among individuals, which will be shown below.

To test the robustness of the non-interior Nash equilibrium in a setting of multiple public goods and to characterize the exact configuration of individual contributions at such an equilibrium, therefore, we assume preferences of individual $i$ to be Cobb-Douglas:

$$
u_{i}\left(c_{i}, X, Y\right)=c_{i} X^{\alpha_{i}} Y^{\beta_{i}}
$$

where $\alpha_{i}, \beta_{i}>0$. Note that this form of the utility function satisfies the assumption made for the utility function (1). In addition, Cobb-Douglas utility functions are weakly separable. As a consequence, individual $i$ 's indifference map in $(X, Y)$ space can be drawn independently of the precise realized value of $c_{i}$ and is homothetic. These features greatly simplify subsequent discussion without making preferences unusual or idiosyncratic in any relevant respect.

Homotheticity of the subutility function in $(X, Y)$ space implies that, for each individual, the locus of points at which the marginal rate of substitution between $X$ and $Y$ equals their relative cost of unity is a straight line passing through the origin. In general, the two individuals' expansion paths in $(X, Y)$ space will have different slopes, as shown in Figure 1. ${ }^{3}$ The separability and homotheticity that are built into our example imply that each

${ }^{3}$ These assumptions rule out the possibility that the two individuals' expansion paths intersect. Consequently, the results we obtain here are valid over the whole $(X, Y)$ space. 
individual $i$ 's most preferred ratio, $(Y / X)_{i}^{*}$, at the given costs is unique. ${ }^{4}$ We will call this ratio individual $i$ 's "ideal ratio." Since the two individuals consume the same quantities of the two public goods, it follows that at any allocation and, a fortiori, at any Nash equilibrium, their marginal rates of substitution between $X$ and $Y$ must differ if $\alpha_{1} / \beta_{1} \neq \alpha_{2} / \beta_{2}$. Panel (a) of Figure 1 shows a situation in which $\alpha_{1} / \beta_{1}>\alpha_{2} / \beta_{2}$, and in Panel (b) $\alpha_{1} / \beta_{1}=\alpha_{2} / \beta_{2}$. Since the situation depicted in Panel (b) to arise is nongeneric, situation (a) therefore seems empirically more plausible.

\subsection{Equilibrium under Cobb-Douglas Preferences}

Without loss of generality, we assume throughout that $\left(\alpha_{1} / \beta_{1}\right)>\left(\alpha_{2} / \beta_{2}\right)$ and therefore $(Y / X)_{1}^{*}<(Y / X)_{2}^{*}$. No allocation, and therefore no equilibrium allocation, equates the marginal rates of substitution across individuals. Moreover, a Nash equilibrium can never imply equilibrium values of $X$ and $Y$ outside the cone spanned by the lines $(Y / X)_{2}^{*}$ and $(Y / X)_{1}^{*}$ in Figure $1(\mathrm{a})$. Denote the Nash equilibrium quantities by $X^{N}$ and $Y^{N}$. For the moment, we leave to one side the precise location of the Nash equilibrium. Our argument to this point implies that there are three possibilities to consider:

1. $Y^{N} / X^{N}=(Y / X)_{1}^{*}$,

2. $(Y / X)_{1}^{*}<Y^{N} / X^{N}<(Y / X)_{2}^{*}$,

3. $Y^{N} / X^{N}=(Y / X)_{2}^{*}$.

Consider each of these in turn:

Case 1: $Y^{N} / X^{N}=(Y / X)_{1}^{*}$ : If the prevailing equilibrium ratio equals individual 1's ideal ratio, then at that allocation $\frac{\partial u_{1} / \partial Y}{\partial u_{1} / \partial X}=1$. This is consistent with individual 1 contributing to both public goods. ${ }^{5}$ However, $\frac{\partial u_{2} / \partial Y}{\partial u_{2} / \partial X}>1$, which implies that individual 2 certainly does not contribute to $X$. At most, he contributes to $Y$ alone. Thus, we can

\footnotetext{
${ }^{4}$ More precisely, the most preferred ratio of individual $i,(Y / X)_{i}^{*}$, at the given costs is obtained from solving the following problem:

$$
\begin{aligned}
& \max _{\left\{c_{i}, X, Y\right\}} u_{i}\left(c_{i}, X, Y\right)=c_{i} X^{\alpha_{i}} Y^{\beta_{i}} \\
& \text { subject to } c_{i}+X+Y=w_{i}+X_{-i}+Y_{-i},
\end{aligned}
$$

where $X_{-i}$ and $Y_{-i}$ are exogenously given. At the optimum, the marginal rate of substitution between the public goods $X$ and $Y$ should be equal to one, that is, $\alpha_{i} Y^{*} / \beta_{i} X^{*}=1$. We can rewrite this condition as the individual $i$ 's most preferred (or ideal) ratio, that is, $(Y / X)_{i}^{*}=\beta_{i} / \alpha_{i}$.

${ }^{5}$ The equality $\frac{\partial u_{1} / \partial Y}{\partial u_{1} / \partial X}=1$ is also consistent with the possibility that individual 1 is contributing to just one of the goods, the tangency between indifference curve and budget line occurring at the point where the quantity of the other good is zero. However, this probability of this event when preferences and income are randomly chosen is equal to zero, and for the sake of simplicity we ignore it.
} 
Table 1: Individual contribution levels in an example with 2 players and 2 public goods

\begin{tabular}{llllll}
\hline Regime & & \multicolumn{1}{c}{$\boldsymbol{x}_{\mathbf{1}}^{N}$} & \multicolumn{1}{c}{$\boldsymbol{y}_{1}^{N}$} & \multicolumn{1}{c}{$\boldsymbol{x}_{2}^{\boldsymbol{N}}$} & \multicolumn{1}{c}{$\boldsymbol{y}_{2}^{\boldsymbol{N}}$} \\
\hline I & $w_{1} \leqq \frac{1}{9}$ & 0 & 0 & $\frac{w w_{2}}{4}$ & $\frac{w_{2}}{2}$ \\
II & $\frac{1}{9}<w_{1} \leqq \frac{1}{3}$ & $\frac{8 w_{1}-w_{2}}{9}$ & 0 & $\frac{3 w_{2}-6 w_{1}}{9}$ & $\frac{4\left(w_{1}+w_{2}\right)}{9}$ \\
III & $\frac{1}{3}<w_{1} \leqq \frac{2}{3}$ & $\frac{2 w_{1}}{3}$ & 0 & 0 & $\frac{2 w_{2}}{3}$ \\
IV & $\frac{2}{3}<w_{1} \leqq \frac{8}{9}$ & $\frac{4\left(w_{1}+w_{2}\right)}{9}$ & $\frac{3 w_{1}-6 w_{2}}{9}$ & 0 & $\frac{8 w_{2}-w_{1}}{9}$ \\
V & $w_{1}>\frac{8}{9}$ & $\frac{w_{1}}{2}$ & $\frac{w_{1}}{4}$ & 0 & 0 \\
\hline
\end{tabular}

conclude that either individual 1 contributes to both goods and individual 2 to $Y$ alone, or individual 1 contributes to both goods and individual 2 to neither.

Now consider the second possibility:

Case 2: $(Y / X)_{2}^{*}>Y^{N} / X^{N}>(Y / X)_{1}^{*}$ : If the equilibrium allocation lies strictly between the two individuals' ideal ratios, then we must have $\frac{\partial u_{2} / \partial Y}{\partial u_{2} / \partial X}>1>\frac{\partial u_{1} / \partial Y}{\partial u_{1} / \partial X}$. Individual 1 contributes only to $X$, and individual 2 contributes only to $Y$.

Case 3: $Y^{N} / X^{N}=(Y / X)_{2}^{*}$ : Analysis of case 3 follows the same lines as that of Case 1.

We need to be more explicit about the precise location of the Nash equilibrium. To do so, we consider a numerical example.

Example 1: The individuals' preferences are represented by the CobbDouglas utility functions:

$$
\begin{aligned}
& u_{1}=c_{1} X^{2} Y, \\
& u_{2}=c_{2} X Y^{2} .
\end{aligned}
$$

Aggregate income in the economy is unity:

$$
w_{1}+w_{2}=1
$$

Elementary manipulations reveal that, depending on the initial income distribution, Nash equilibrium may fall into any one of five regimes, according to the pattern of individual contributions. Table 1 summarizes the regimes.

A significant feature of the table is the following: in Regimes II and IV, the aggregate quantities of the two public goods depend only on aggregate income, not on its precise distribution: when $1 / 9<w_{1} \leqq 1 / 3$,

$$
X^{N}=\frac{2\left(w_{1}+w_{2}\right)}{9}=\frac{2}{9} \text { and } Y^{N}=\frac{4\left(w_{1}+w_{2}\right)}{9}=\frac{4}{9},
$$




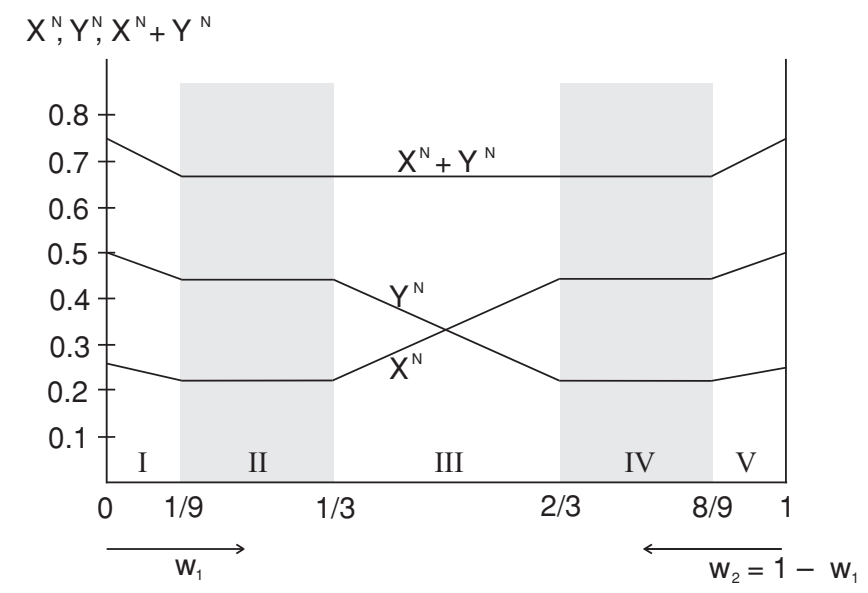

Figure 2: The provision levels of the public goods $X$ and $Y$ at a Nash equilibrium with respect to the distribution of income.

while when $2 / 3<w_{1} \leqq 8 / 9$,

$$
X^{N}=\frac{4\left(w_{1}+w_{2}\right)}{9}=\frac{4}{9} \quad \text { and } \quad Y^{N}=\frac{2\left(w_{1}+w_{2}\right)}{9}=\frac{2}{9} .
$$

Figure 2 illustrates the behavior of equilibrium provision of the public goods as the income distribution varies.

It may be confirmed that when $1 / 9<w_{1} \leqq 1 / 3$,

$$
\frac{\partial u_{2} / \partial X}{\partial u_{2} / \partial c_{2}}=\frac{c_{2}}{X}=1>\frac{1}{4}=\frac{c_{1}}{Y}=\frac{\partial u_{1} / \partial Y}{\partial u_{1} / \partial c_{1}} .
$$

Similarly, when $2 / 3<w_{1} \leqq 8 / 9$,

$$
\frac{\partial u_{2} / \partial X}{\partial u_{2} / \partial c_{2}}=\frac{c_{2}}{X}=\frac{1}{4}<1=\frac{c_{1}}{Y}=\frac{\partial u_{1} / \partial Y}{\partial u_{1} / \partial c_{1}} .
$$

Thus, income distributions within these ranges certainly do not generate constrained Pareto-efficient equilibria. In fact, in this example there are just three constrained Pareto-efficient equilibrium allocations. Two occur at extreme distributions: $\left(w_{1}, w_{2}\right)=(0,1)$ and $\left(w_{1}, w_{2}\right)=(1,0)$. These are constrained Pareto efficient for a familiar reason: if one individual enjoys all the income, there is no way to compensate her for any perturbation away from her preferred public good mix, since the other individual's endowment is zero. The third constrained Pareto-efficient equilibrium allocation is more interesting, and arises when the initial distribution is equal: $w_{1}=w_{2}=1 / 2$.

The source of the constrained inefficiency is readily explained. In the numerical example, suppose that $\left(w_{1}, w_{2}\right)=(2 / 3,1 / 3)$. Constrained Pareto 
efficiency requires

$$
\frac{\partial u_{1} / \partial X}{\partial u_{1} / \partial c_{1}}+\frac{\partial u_{2} / \partial X}{\partial u_{2} / \partial c_{2}}=\frac{\partial u_{1} / \partial Y}{\partial u_{1} / \partial c_{1}}+\frac{\partial u_{2} / \partial Y}{\partial u_{2} / \partial c_{2}}
$$

At the Nash equilibrium associated with an income distribution in Regime IV, we know that $\frac{\partial u_{1} / \partial X}{\partial u_{1} / \partial c_{1}}=\frac{\partial u_{1} / \partial Y}{\partial u_{1} / \partial c_{1}}=\frac{\partial u_{2} / \partial Y}{\partial u_{2} / \partial c_{2}}=1$. Thus constrained efficiency requires that

$$
\frac{\partial u_{2} / \partial X}{\partial u_{2} / \partial c_{2}}=1
$$

However, this cannot hold at this allocation, at which the ratio $X / Y$ is far from individual 2's ideal ratio. Intuitively, a reduction in $X$, accompanied by an increase in $Y$ that keeps $X+Y$ constant, has only a second-order adverse effect on individual 1's utility. However, it has a first-order beneficial effect on individual 2's utility. There is a transfer of private good consumption from individual 2 to 1 , which, if it were to accompany the substitution of $Y$ for $X$, would make both better off than at the initial equilibrium.

Starting at $\left(w_{1}, w_{2}\right)=(2 / 3,1 / 3)$, consider the path traced out by the resulting equilibria as individual 1's initial income falls and that of individual 2 rises. The associated equilibrium involves less $X$ and more $Y$, while $X+Y$ remains constant (until we reach the point where $\left.\left(w_{1}, w_{2}\right)=(1 / 3,2 / 3)\right)$. As we move along the path traced out by the resulting equilibria within Regime III, it remains the case that $\frac{\partial u_{1} / \partial X}{\partial u_{1} / \partial c_{1}}=\frac{\partial u_{2} / \partial Y}{\partial u_{2} / \partial c_{2}}=1$. However, $\frac{\partial u_{1} / \partial Y}{\partial u_{1} / \partial c_{1}}$ falls and $\frac{\partial u_{2} / \partial X}{\partial u_{2} / \partial c_{2}}$ rises. Thus, there is one, and only one, Nash equilibrium on the path that is constrained Pareto efficient. This is the symmetric allocation at which $(X, Y)=(1 / 3,1 / 3)$, achieved from an initial income distribution of $\left(w_{1}, w_{2}\right)=(1 / 2,1 / 2)$.

Hence, we can summarize the conclusions of the 2-individual CobbDouglas example as follows: If the individuals' ideal ratios of public goods differ (i.e., $\alpha_{1} / \beta_{1} \neq \alpha_{2} / \beta_{2}$ ), then (i) there is no Nash equilibrium at which both individuals contribute to both public goods and (ii) there is only one interior initial income distribution for which the associated Nash equilibrium is constrained Pareto efficient.

Several of the results under Cobb-Douglas preferences (12) remain valid under more general preferences (1); such as the extreme distributions $\left(w_{1}, w_{2}\right)=(0,1)$ and $\left(w_{1}, w_{2}\right)=(1,0)$ realize constrained Pareto-efficient allocations; in a range of income distribution which is consistent with neutrality, i.e., either $\frac{\partial u_{1} / \partial X}{\partial u_{1} / \partial c_{1}}=\frac{\partial u_{2} / \partial X}{\partial u_{2} / \partial c_{1}}=\frac{\partial u_{2} / \partial Y}{\partial u_{2} / \partial c_{2}}=1$ or $\frac{\partial u_{1} / \partial X}{\partial u_{1} / \partial c_{1}}=\frac{\partial u_{1} / \partial Y}{\partial u_{1} / \partial c_{1}}=\frac{\partial u_{2} / \partial Y}{\partial u_{2} / \partial c_{2}}=$ 1 holds, the resulting Nash equilibrium allocation is not constrained Pareto efficient and so on. 


\section{Neutrality in a $2 \times 2$ Model}

We turn now to the matter of neutrality. Figure 2 shows two shaded regions, corresponding to Regimes II and IV, in which income transfers do not affect the real equilibrium (i.e., the levels of $X^{N}$ and $Y^{N}$ ).

Note that in Regime II both individuals make positive contributions to public good $X$-we will say that, in that regime, they "share an active interest" in $X$. More formally, we define sharing an active interest as follows:

DEFINITION 2: Individuals $i$ and $j$ share an active interest in public good $k$ at an allocation if, at that allocation, both individuals' marginal rates of substitution between that public good and the private good equal the relative cost ratio, $p_{k} / p_{c}$, where $p_{k}$ represents the unit cost of producing public good $k$.

Similarly, in Regime IV they share an active interest in $Y$. Suppose that the initial income distribution places the individuals somewhere in Regime II. Now transfer an amount $\Delta$ from individual 1 and give it to individual 2.

Denote the initial equilibrium allocation by the quantities

$$
\left(c_{1}^{0}, c_{2}^{0}, x_{1}^{0}, x_{2}^{0}, y_{1}^{0}, y_{2}^{0}\right),
$$

where, by assumption, $y_{1}^{0}=0$. Such an allocation implies satisfaction of both individuals' budget constraints with equality:

$$
c_{i}^{0}+x_{i}^{0}+y_{i}^{0}=w_{i}, i=1,2 .
$$

After the transfer, consider the following allocation:

$$
\left(c_{1}^{0}, c_{2}^{0}, x_{1}^{0}-\Delta, x_{2}^{0}+\Delta, y_{1}^{0}, y_{2}^{0}\right) .
$$

If $\Delta \leqq x_{1}^{0}$, such an allocation is feasible, and satisfies both individuals' budget constraints with equality:

$$
\begin{aligned}
& c_{1}^{0}+\left(x_{1}^{0}-\Delta\right)+y_{1}^{0}=w_{1}-\Delta, \\
& c_{2}^{0}+\left(x_{2}^{0}+\Delta\right)+y_{2}^{0}=w_{2}+\Delta .
\end{aligned}
$$

Moreover, each individual is still enjoying the same real consumption bundle, since neither of the private consumption levels changed, nor have either of the total provision levels of the public goods. Since the relative costs are, by assumption, constant, the first-order conditions characterizing a Nash equilibrium allocation remain unaffected. Thus, this allocation remains a Nash equilibrium. The inequality $\Delta \leqq x_{1}^{0}$ ensures that the new allocation remains within Regime II.

Note that this proposition is not confined to the Cobb-Douglas preferences of our example, because the above proof for neutrality does not depend on the form of utility functions. Thus, although in general we cannot have both individuals contributing to both goods, the following proposition establishes the continued relevance of the neutrality proposition: 
PROPOSITION 3: In the $2 \times 2$ model with preferences described by (1), a shared active interest in just one public good is sufficient to generate neutrality, as long as the income redistribution is "small" in the sense that no individual gives up an amount of income in excess of his or her initial individual contribution level.

\section{Constrained Pareto Efficiency and Neutrality in a $3 \times 2$ Model}

The introduction of a third individual enables us to check the robustness of the constrained Pareto efficiency and neutrality properties of the twoindividual game, and also uncovers an additional possibility-that of "partial neutrality." We continue to assume that there are two public goods. We call this the $3 \times 2$ model. Assume that the individuals can be strictly ranked according to their ideal ratios at the prevailing costs:

$$
\left(\frac{Y}{X}\right)_{1}^{*}>\left(\frac{Y}{X}\right)_{2}^{*}>\left(\frac{Y}{X}\right)_{3}^{*}
$$

Again, a specific Cobb-Douglas example enables us to locate various regimes, distinguished by the patterns of positive and zero contributions. Let the three individuals have the following preferences:

$$
\begin{aligned}
& u_{1}=c_{1} X^{3} Y, \\
& u_{2}=c_{2} X^{2} Y^{2}, \\
& u_{3}=c_{3} X Y^{3} .
\end{aligned}
$$

The total income of the group is unity, and unit production costs are all unity. Figure 3 depicts all possible income distributions using the two-dimensional simplex - see the inset for clarification of our convention for depicting initial incomes.

Each point in the simplex represents an income distribution among three individuals, the individuals' incomes being the lengths of that point's perpendiculars to the three sides. Tedious calculations allow us to identify the 17 regimes that exist in this example. Figure 3 shows the 17 regimes. In each, the listed variables $x_{i}, y_{i}$ indicate the contribution levels that are positive in that regime. The stated equalities involving the income levels hold at the indicated boundaries between regimes. Figure 4 reproduces the regimes appearing in Figure 3 and introduces our labeling conventions. The shading will be explained below.

\subsection{Neutrality in a $3 \times 2$ Model}

At any income distribution within Regime $\mathrm{C}$ in Figure 4, individuals 1 and 2 share an active interest in $X$, and individuals 2 and 3 share an active interest in $Y$. Thus, equilibrium is unaffected by any set of transfers across individuals that keeps the distribution within Regime C (we call this neutrality property 


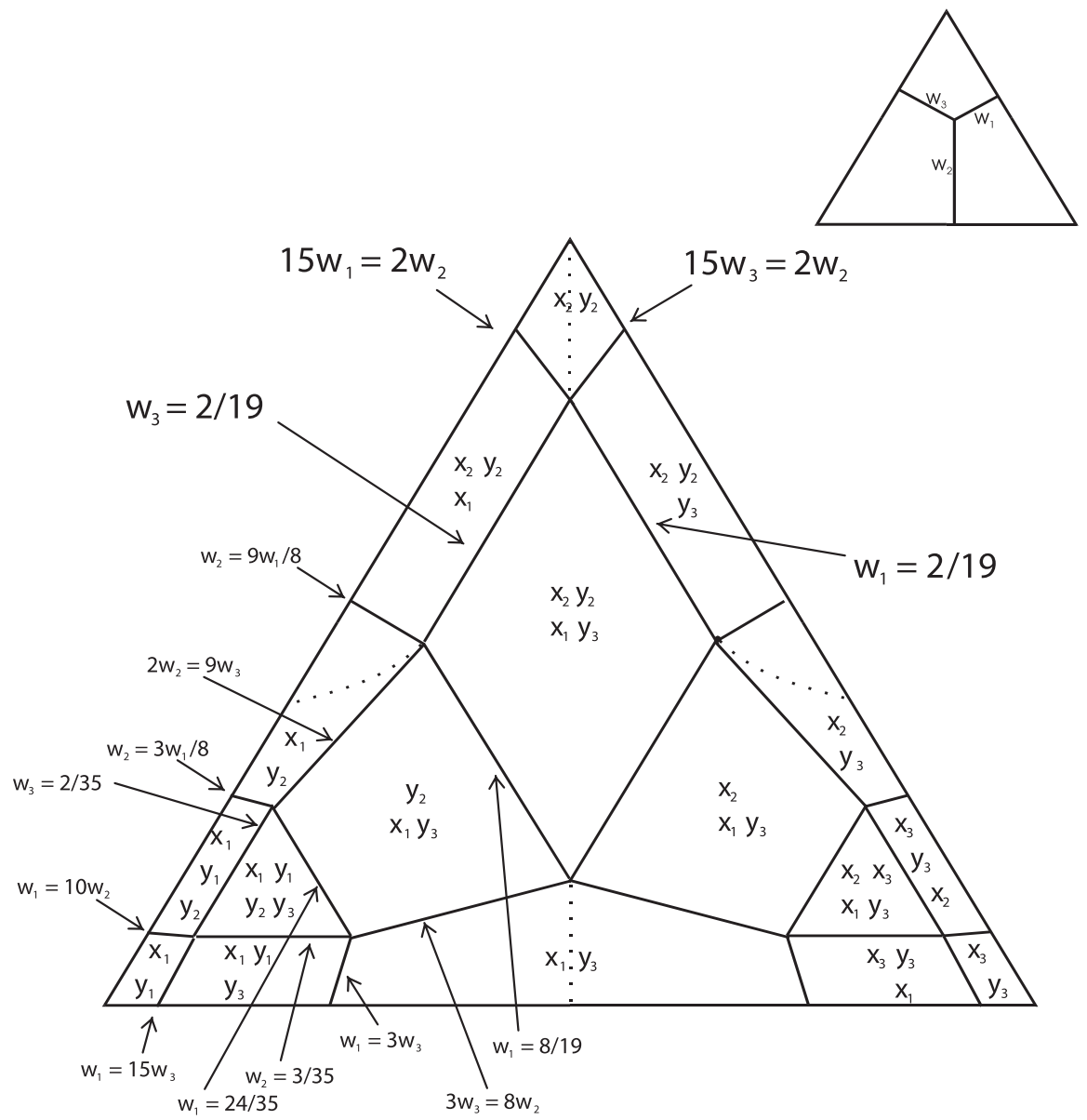

Figure 3: The configuration of positive contributions made by individuals 1,2 , and 3 with respect to a distribution of income.

"full neutrality"). The same observation holds for any pair of income distributions that lie within Regime J, and for any pair within K.

Within each of Regimes B, G, and I, individuals 1 and 2 share an active interest in either $X$ or $Y$. However, neither of these individuals has a shared active interest with individual 3. Within each of these Regimes, although "full neutrality" fails to hold, there is a "partial neutrality" property, which will be defined below. Suppose we start at an equilibrium within Regime B. After any transfer between the two individuals with a shared interest that remains within Regime B, that equilibrium remains undisturbed. However, any net transfer between individual 3 and either of the other two individuals will lead to an equilibrium in a different regime. The income transfer must keep $w_{3}$, and therefore the sum $w_{1}+w_{2}$, constant if it is to produce an unchanged 


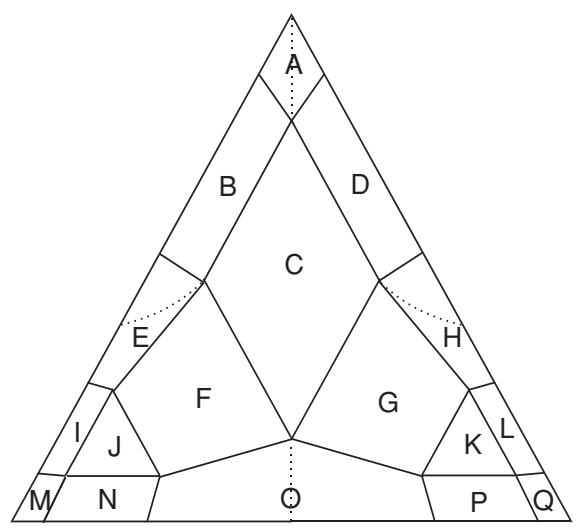

Figure 4: Labels of regimes identified in Figure 3.

equilibrium. Again, both the initial and the final income distributions must lie within the same regime. Hence, for example, a shift from a point in B to a point in $\mathrm{G}$ will not lead to neutrality.

In the same way, areas D, F, and L represent regimes within which there is partial neutrality with respect to transfers between individuals 2 and 3, but not involving individual 1 . Finally, areas $\mathrm{N}$ and $\mathrm{P}$ represent regimes for which there is partial neutrality between individuals 1 and 3 .

\subsection{Pareto (In)efficiency in the $3 \times 2$ Example}

We now show that, within the context of our specific numerical example, the set of initial income distributions that lead to constrained Pareto-efficient equilibria is the set represented by (i) all the points within Regime C, (ii) those points within Regime A at which $w_{1}=w_{3}$, (iii) those points within Regime $\mathrm{O}$ at which $w_{1}=w_{3}$, and (iv) points along the dotted lines in the Regimes $\mathrm{E}$ and $\mathrm{H}$ in Figures 3 and 4.

First, consider Regime C. Within this regime, we know that the following conditions hold:

$$
\frac{\partial u_{1} / \partial X}{\partial u_{1} / \partial c_{1}}=\frac{\partial u_{2} / \partial X}{\partial u_{2} / \partial c_{2}}=\frac{\partial u_{2} / \partial Y}{\partial u_{2} / \partial c_{2}}=\frac{\partial u_{3} / \partial Y}{\partial u_{3} / \partial c_{3}}=1 .
$$

We also know that

$$
\begin{gathered}
3 c_{1}=x_{1}+x_{2}=X, \quad 2 c_{2}=x_{1}+x_{2}=X, \\
2 c_{2}=y_{2}+y_{3}=Y, \quad 3 c_{3}=y_{2}+y_{3}=Y, \\
x_{3}=y_{1}=0 .
\end{gathered}
$$


Constrained Pareto efficiency requires that

$$
\frac{\partial u_{1} / \partial X}{\partial u_{1} / \partial c_{1}}+\frac{\partial u_{2} / \partial X}{\partial u_{2} / \partial c_{2}}+\frac{\partial u_{3} / \partial X}{\partial u_{3} / \partial c_{3}}=\frac{\partial u_{1} / \partial Y}{\partial u_{1} / \partial c_{1}}+\frac{\partial u_{2} / \partial Y}{\partial u_{2} / \partial c_{2}}+\frac{\partial u_{3} / \partial Y}{\partial u_{3} / \partial c_{3}},
$$

or, in view of (13),

$$
\frac{\partial u_{3} / \partial X}{\partial u_{3} / \partial c_{3}}=\frac{\partial u_{1} / \partial Y}{\partial u_{1} / \partial c_{1}} .
$$

Differentiating the utility functions, this requires that

$$
\frac{c_{3}}{X}=\frac{c_{1}}{Y},
$$

which is clearly implied by (14). Thus, all allocations in Regime C are constrained Pareto efficient.

Now consider Regime A. The pattern of positive contributions implies that, at any allocation in $\mathrm{A}, \frac{\partial u_{2} / \partial X}{\partial u_{2} / \partial c_{2}}=\frac{\partial u_{2} / \partial Y}{\partial u_{2} / \partial c_{2}}=1$. This, in turn, implies

$$
\begin{gathered}
2 c_{2}=x_{2}=X=\frac{2 w_{2}}{5}, \\
2 c_{2}=y_{2}=Y=\frac{2 w_{2}}{5}, \\
x_{1}=x_{3}=y_{1}=y_{3}=0, \\
c_{1}=w_{1}, \\
c_{3}=w_{3} .
\end{gathered}
$$

Differentiating the utility functions, the requirement for constrained Pareto efficiency becomes

$$
\frac{3 c_{1}}{X}+\frac{c_{3}}{X}=\frac{c_{1}}{Y}+\frac{3 c_{3}}{Y} .
$$

Substituting from the system (15), constrained Pareto efficiency requires that

$$
\frac{3 w_{1}}{2 w_{2} / 5}+\frac{w_{3}}{2 w_{2} / 5}=\frac{w_{1}}{2 w_{2} / 5}+\frac{3 w_{3}}{2 w_{2} / 5},
$$

which in turn requires that

$$
w_{1}=w_{3} .
$$

A similar analysis reveals that within Regime A, those income distributions in which $w_{1}=w_{3}$ yield constrained Pareto-efficient equilibrium outcomes.

Now consider Regime H. Here $x_{2}$ and $y_{3}$ are the only nonzero contributions. All others are zero. Thus, we know that

$$
\frac{\partial u_{2} / \partial X}{\partial u_{2} / \partial c_{2}}=\frac{\partial u_{3} / \partial Y}{\partial u_{3} / \partial c_{3}}=1
$$


In the Cobb-Douglas example, condition (16) is reduced to

$$
\frac{\partial u_{2} / \partial X}{\partial u_{2} / \partial c_{2}}=\frac{2 c_{2}}{X}=1 \quad \text { and } \quad \frac{\partial u_{3} / \partial Y}{\partial u_{3} / \partial c_{3}}=\frac{3 c_{3}}{Y}=1,
$$

which, respectively, imply

$$
2 c_{2}=X=\frac{2 w_{2}}{3} \quad \text { and } \quad 3 c_{3}=Y=\frac{3 w_{3}}{4} .
$$

Also, note in passing that

$$
c_{1}=w_{1} .
$$

In view of (16), constrained Pareto efficiency requires that

$$
\frac{\partial u_{1} / \partial X}{\partial u_{1} / \partial c_{1}}+\frac{\partial u_{3} / \partial X}{\partial u_{3} / \partial c_{3}}=\frac{\partial u_{1} / \partial Y}{\partial u_{1} / \partial c_{1}}+\frac{\partial u_{2} / \partial Y}{\partial u_{2} / \partial c_{2}} .
$$

In the Cobb-Douglas example, the only allocations at which this condition is in fact satisfied are those at which

$$
\frac{3 c_{1}}{X}+\frac{c_{3}}{X}=\frac{c_{1}}{Y}+\frac{2 c_{2}}{Y} .
$$

Using the solutions for these quantities that hold in Regime $\mathrm{H}$, constrained Pareto efficiency requires that

$$
\frac{3 w_{1}}{2 w_{2} / 3}+\frac{w_{3} / 4}{2 w_{2} / 3}=\frac{w_{1}}{3 w_{3} / 4}+\frac{2 w_{2} / 3}{3 w_{3} / 4} .
$$

Substituting the condition $w_{1}+w_{2}+w_{3}=1$ for $w_{1}$ in the above expression, and rearranging, the locus of constrained Pareto efficiency within Regime $\mathrm{H}$ is given by

$$
\frac{2}{3} w_{2}-\frac{9}{4} w_{3}+\frac{19}{12} w_{2} w_{3}-\frac{2}{9} w_{2}^{2}+\frac{33}{16} w_{3}^{2}=0 .
$$

A symmetric requirement, with the subscripts "2" and "3" reversed, holds within Regime E.

Within the remaining regimes, it may be confirmed that the equilibrium allocations cannot be constrained Pareto efficient.

We end this subsection with a warning. The constrained Pareto efficiency of the set of equilibrium allocations within Regime $\mathrm{C}$ is not robust to changes in parameters. Our numerical example has a very symmetric structure, which is responsible for the positive mass set of constrained Pareto-efficient equilibria. Consider the family of examples in which the individual preferences are

$$
\begin{aligned}
& u_{1}=c_{1} X^{3+\epsilon} Y, \\
& u_{2}=c_{2} X^{2} Y^{2}, \\
& u_{3}=c_{3} X Y^{3+\eta} .
\end{aligned}
$$


By continuity, Regime $\mathrm{C}$ continues to have positive measure for small values of $\varepsilon$ and $\eta$. If we are in Regime $\mathrm{C}$, then

$$
\begin{aligned}
(3+\varepsilon) c_{1} & =x_{1}+x_{2}=X, \\
2 c_{2} & =x_{1}+x_{2}=X, \\
2 c_{2} & =y_{1}+y_{2}=Y, \\
(3+\eta) c_{3} & =y_{1}+y_{2}=Y, \\
x_{3} & =y_{1}=0 .
\end{aligned}
$$

Constrained Pareto efficiency requires that

$$
\frac{\partial u_{3} / \partial X}{\partial u_{3} / \partial c_{3}}=\frac{\partial u_{1} / \partial Y}{\partial u_{1} / \partial c_{1}} \quad \text { or } \quad \frac{c_{3}}{X}=\frac{c_{1}}{Y}
$$

which is rearranged as

$$
\frac{c_{3}}{c_{1}}=\frac{X}{Y} .
$$

Looking at the equilibrium conditions within Regime C, (20) and (17) together imply

$$
\frac{c_{3}}{c_{1}}=\frac{Y}{X} \frac{(3+\varepsilon)}{(3+\eta)}=\frac{(3+\varepsilon)}{(3+\eta)},
$$

where the last equality follows from (18) and (19). Clearly, the equilibrium associated with Regime $\mathrm{C}$ does not satisfy the requirements for constrained Pareto efficiency except when $\varepsilon=\eta$.

We conclude that, within the restricted class of Cobb-Douglas preferences, if the parameters that represent those preferences are independent draws from some population, the probability that the resulting equilibrium generates a constrained Pareto-efficient equilibrium is zero. In general, we can expect equilibrium in a model with two or more public goods to generate the "wrong" mix of public goods.

\section{The $n \times m$ Model}

In this section, we briefly discuss two directions in which our results may be extended. First, we consider models with many individuals and many public goods. We then address the issue of what can be said when preferences are rather more general. We argue that the main role of our restrictions on preferences is to limit the number of possible regimes-that is, patterns of positive contributions across individuals-and to facilitate the computation of these regimes from knowledge of the preferences and endowments. For 
example, relaxation of homotheticity destroys our ability to rank individuals globally according to their ideal ratios. The situation is analogous to factor intensity rankings of industries in international trade theory.

We have already introduced the idea of two individuals sharing an active interest in a public good. We now introduce the idea of individuals being linked in a model with potentially many public goods and players.

DEFINITION 3: Individuals $h$ and $h+k$ are linked at an equilibrium if there is a set of public goods (labeled $\} G_{1}, G_{2}, \ldots, G_{k}$ ) and a set of individuals (labeled $h, h+1, \ldots, h+k)$ such that, at that equilibrium,

- Individual $h$ shares an active interest with individual $h+1$ in public good $G_{1}$,

- Individual $h+1$ shares an active interest with individual $h+2$ in public $\operatorname{good} G_{2}$,

- $\ldots$,

- Individual $h+k-1$ shares an active interest with individual $h+k$ in public $\operatorname{good} G_{k}$.

Clearly, if individuals $h$ and $h+k$ are linked, so too are any two individuals belonging to the chain that links them. Now consider a Nash equilibrium in an economy consisting of many individuals, a single private good, and many public goods. Suppose that individuals $h$ and $h+k$ are linked. There is no loss of generality in supposing that they are linked through individuals $h+1, h+2, \ldots, h+k-1$, and by public goods $1,2, \ldots, k$. We therefore have the following chain of relationships consisting of their budget constraints at equilibrium:

$$
\begin{aligned}
c_{h}^{0}+g_{h 1}^{0} & =w_{h}, \\
c_{h+j}^{0}+g_{h+j j}^{0}+g_{h+j j+1}^{0} & =w_{h+j} \quad j=1,2, \ldots, k-1, \\
c_{h+k}^{0}+g_{h+k k}^{0} & =w_{h+k},
\end{aligned}
$$

where $g_{h+j}^{0}$ stands for individual $h+j$ 's contribution to public good $j$ at the initial equilibrium, and we assume that all the individual contribution levels are strictly positive in the chain. ${ }^{6}$ Now suppose that there is a redistribution of income from individual $h$ to individual $h+k$, so that their incomes become $w_{h}-\Delta$ and $w_{h+k}+\Delta$, respectively. Now consider the new allocationin which the quantities are indicated by the superscript $1-$ characterized by

\footnotetext{
${ }^{6}$ Individual $j$ may make positive contributions to other public goods in addition to the public goods $j$ and $j+1$ as well. For expositional simplicity, we have assumed that individual $j$ makes contributions only to the public goods $j$ and $j+1$.
} 
the following:

$$
\begin{aligned}
\left(c_{h}^{1}, g_{h 1}^{1}\right) & =\left(c_{h}^{0}, g_{h 1}^{0}-\Delta\right), \\
\left(c_{h+j}^{1}, g_{h+j j}^{1}, g_{h+j j+1}^{1}\right) & =\left(c_{h+j}^{0}, g_{h+j j}^{0}+\Delta, g_{h+j j+1}^{0}-\Delta\right), j=1,2, \ldots, k-1, \\
\left(c_{h+k}^{1}, g_{h+k k}^{1}\right) & =\left(c_{h+k}^{0}, g_{h+k k}^{0}+\Delta\right) .
\end{aligned}
$$

If $\Delta \leqq \min \left\{g_{h 1}^{0}, g_{h+12}^{0}, \ldots, g_{h+k-1 k}^{0}\right\}$, the new allocation is feasible in the sense that the budget constraint of every individual remains satisfied with equality. Since each of the individuals involved is enjoying an unchanged consumption of the private good (i.e., $c_{i}^{0}=c_{i}^{1}, i=h, h+1, \ldots, h+k$ ), and an unchanged aggregate level of all public goods (i.e., $G_{j}^{0}=G_{j}^{1}, j=1,2, \ldots, k$ ), and since each has unchanged preferences, the first-order conditions associated with each contributor's maximization problem remain satisfied, and the allocation therefore remains a Nash equilibrium.

PROPOSITION 4 (Partial neutrality): An income redistribution that is restricted to a set of linked individuals, and that maintains the links between them, has no effect on the original equilibrium allocation.

This slightly extends Theorem 7 in Bergstrom, Blume, and Varian (1986). They consider three groups of individuals-those who contribute "only to G," those who contribute "to G and $\mathrm{H}$," and those who contribute "only to $\mathrm{H}$," where $\mathrm{G}$ and $\mathrm{H}$ represent public goods. The statement of their theorem requires that the income transfers leave unchanged the total income of each of these three groups. However, a net transfer that reduces the wealth of the first group and increases that of the last group is also neutral provided that the initial members of the three groups remain linked. ${ }^{7}$

Remark 1: Even under more general preferences such as (1), if we observe the pattern of positive contributions at a given equilibrium, this information alone allows us to make inferences about whether neutrality characterizes the observed equilibrium. This can be done by inspecting whether or not a redistribution of income is limited within the subset of linked individuals in the sense of Definition 3. However, we have argued that, although the possibility of an equilibrium that is constrained

\footnotetext{
${ }^{7}$ To see this, suppose there are just three individuals. The initial Nash equilibrium is $\left(G^{0}, H^{0}\right)$. Suppose individual GONLY, who contributes only to G, loses income $\triangle w_{G O N L Y}$. HONLY's income rises by the same amount. Such a transfer is not allowed by Bergstrom, Blume, and Varian (1986). Now consider the allocation in which $\Delta g_{G O N L Y}=\Delta w_{G O N L Y}, \Delta h_{H O N L Y}=\Delta w_{H O N L Y}=-\Delta w_{G O N L Y}$, and $\left(\Delta g_{B O T H}, \Delta g_{B O T H}\right)=$ $\left(-\Delta w_{G O N L Y},-\Delta h_{H O N L Y}\right)$. A quick check shows that, at this new equilibrium, $G^{1}=G^{0}+$ $\Delta g_{G O N L Y}+\Delta g_{B O T H}=G^{0}, H^{1}=H^{0}+\Delta h_{H O N L Y}+\Delta h_{B O T H}=H^{0}$, and all three individuals enjoy an unchanged level of private good consumption. It is also an equilibrium. Thus, a net transfer between two linked individuals, provided it does not destroy the chain that links them, preserves the initial allocation as an equilibrium.
} 
Pareto efficient cannot be completely ruled out, equilibrium will generically imply an inefficient mix of the public goods.

There seems little to be gained by exploring these complications further. It is sufficient to observe that, at any observed equilibrium, we know the pattern of observed contributions and therefore can identify the regime in which the equilibrium is located.

\section{Conclusions}

Although several papers formally incorporate the possibility of many public goods, little attention has been paid to the new questions that one can ask of equilibrium in a world of many public goods. We have elucidated the neutrality properties of such models, and have shown that the presence of two or more public goods introduces a new aspect to the consideration of the inefficiency of equilibrium. Not only may too few resources be devoted to public good production, but their mix may also be inefficient. We claim that this last property is generic.

We have not explored the policy implications of the multiple public good model extensively. It may be observed that there are many public goods in the real world, some supplied exclusively by governments, some by private agents, and some by both. This observation provokes several interesting questions. For example, why do governments choose to supply the particular goods they do? Which public goods should be supplied by government? Might the government's attempt to augment provision of a particular public good create inefficiency by exacerbating the inefficient mix problem? The existing literature on private provision of public goods, based as it is on a single public good model, cannot address such issues. The analysis of this paper is a first step toward addressing these more interesting and important issues in a multiple public good setting.

\section{References}

BERGSTROM, T. C., L. BLUME, and H. VARIAN (1986) On the private provision of public goods, Journal of Public Economics 29, 25-49.

CORNES, R. C., and T. SANDLER (1985) The simple analytics of pure public good provision, Economica 52, 103-116.

CORNES, R. C., and A. S. SCHWEINBERGER (1996) Free riding and the inefficiency of the private production of pure public goods, Canadian Journal of Economics $\mathbf{2 9}$, 70-91.

DASGUPTA, I., and R. KANBUR (2005) Bridging communal divides: Separation, patronage and integration, in The Social Economics of Poverty: On Identities, Groups, Communities and Networks, C. Barrett ed. London: Routledge.

KEMP, M. C. (1984) A note on the theory of international transfers, Economics Letters 14, 259-262. 